\title{
Analysis and simulations of a frequency synthesizer with internal and external noise sources
}

\author{
G. S. Sangha and M. H. W. Hoffmann \\ Department of Microwave Techniques, University of Ulm, Germany
}

\begin{abstract}
In this paper, a novel technique to analyse the noise behaviour of a second-order-PLL based synthesizer is demonstrated. Its non-linear behaviour is analyzed with independent noise-sources introduced at different points of the circuit. Phase plane trajectories will be used to demonstrate that noise sources at different locations in the loop produce qualitatively different contributions to the output signal of the synthesizer.
\end{abstract}

\section{Introduction}

The PLL based synthesizer has become an important component for a wide range of communications systems. The increasing requirements concerning stability of synthesizers are requiring deeper insight into the non-linear effects of different noise sources in these systems. These affect the phase noise of the output signal. Previous papers have been published on different aspects of phase noise in synthesizer signals, (e.g. Kundert, 2001; Curtin and O'Brien, 1999; Heydari, 2004; Sancho and Suàrez, 2001; Lee and Hajimiri, 2000; Blanchard, 1976; Drucker, 2000), yet a systematic overview on the effects of different noise sources on the phase noise and its spectral forming by non-linearities is still missing. This paper attempts to contribute to close that gap.

\section{Analysis methodology}

A PLL based frequency synthesizer is used for the analysis. It is shown in Fig. 1 as a model in terms of phases. In order to take into consideration a more complete set of parameters influencing the noise behaviour of the synthesizer, also the fluctuations of the reference-signal amplitude $R$ and localoscillator amplitude $n_{4}$ are considered in the phase detector

Correspondence to: M. H. W. Hoffmann

(michael.hoffmann@ieee.org) function $f\left(R, n_{4}, \varphi\right)$. With these terms, the PLL might be described by the following non-linear differential equation:

$$
\begin{aligned}
\tau_{1} \ddot{\varphi} & +\left\{b+K_{o} \tau_{2} \frac{\partial f\left(R, n_{4}, \varphi\right)}{\partial \varphi}\right\} \dot{\varphi}+K_{o} f\left(R, n_{4}, \varphi\right) \\
& +K_{o} \tau_{2} \frac{\partial f\left(R, n_{4}, \varphi\right)}{\partial R} \dot{R}+K_{o} \tau_{2} \frac{\partial f\left(R, n_{4}, \varphi\right)}{\partial n_{4}} \dot{n}_{4} \\
& =\tau_{1} \ddot{\theta}_{i}+b \dot{\theta}_{i}-b \omega_{o}
\end{aligned}
$$

In this equation, $\varphi$ is the phase difference between the reference-phase $\theta_{i}$ and the local-oscillator phase $\theta_{o}$.

In this paper a non-standard noise modelling technique following Rice $(1944,1945)$ will be used.

If a linear analysis is sufficient, the phase detector function might be linearised using a first order Taylor series approach. This leads to a linear differential equation, which is different from what is found in literature. This is due to the above mentioned introduction of new sources of noise, which are usually not taken into account. A closer analysis of the linearised behaviour has been presented by the authors in Sangha and Hoffmann (2005).

The noise sources considered in the following analysis are the following:

- The noise at input of the PLL including phase noise as well as amplitude fluctuations

- The amplitude variations of the local oscillator, since they influence the phase-detector output

- Noise source included in the phase-detector, and in the control filter.

\subsection{Rice's noise models}

Rice $(1944,1945)$ has developed two different models to describe the measured noise mathematically. The first model is 


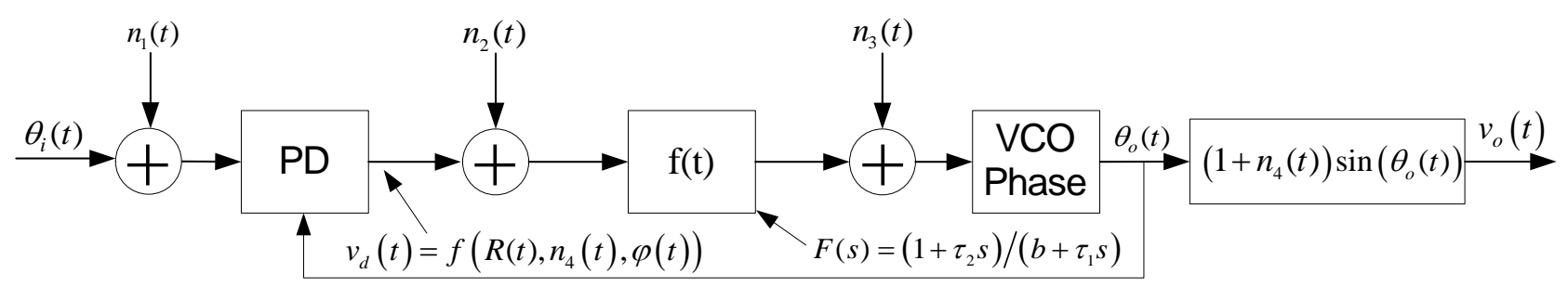

Fig. 1. Synthesizer model used for the analysis
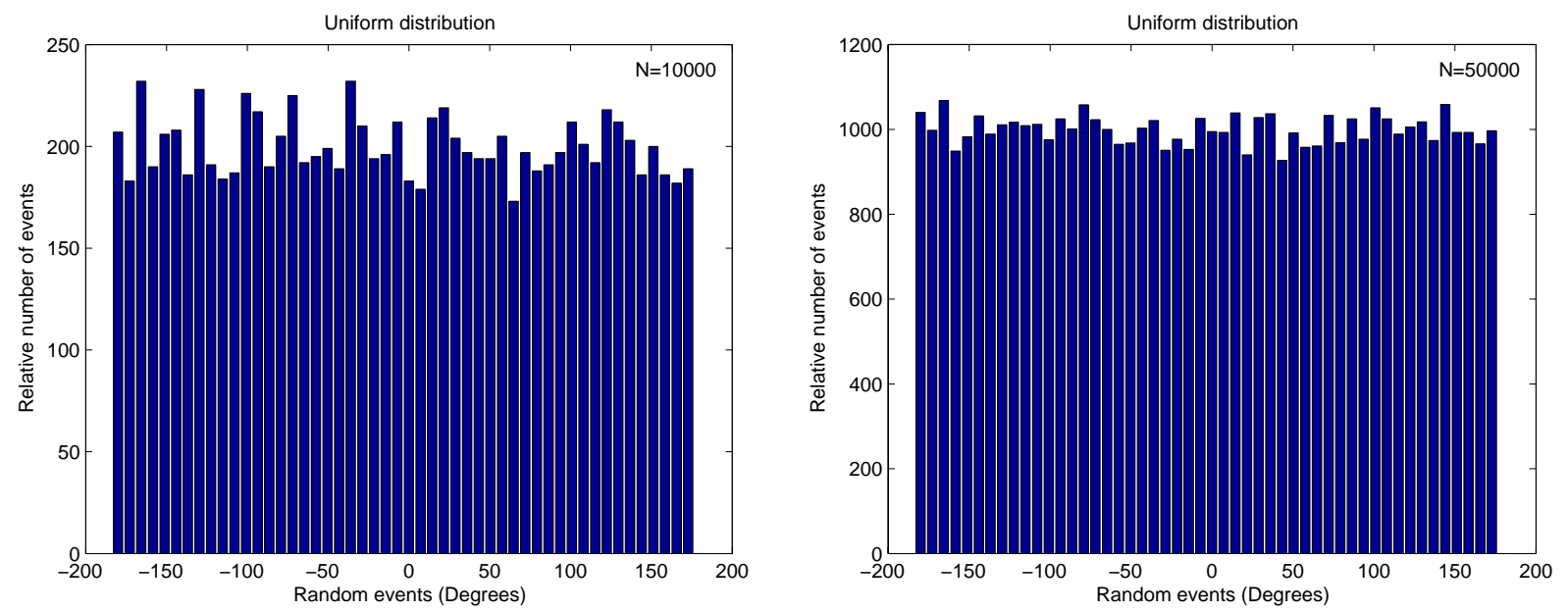

Fig. 2. Uniform distribution for different number of events.

well known and widely used:

$$
\begin{aligned}
u(t) & =V_{0} / 2+\sum_{n=1}^{N}\left\{V_{n} \cos \left(n \Delta \omega t+\varphi_{n}\right)\right\} \\
& =A_{0} / 2+\sum_{n=1}^{N}\left\{A_{n} \cos (n \Delta \omega t)+B_{n} \sin (n \Delta \omega t)\right\}
\end{aligned}
$$

In this model both $V_{n}$ and $\varphi_{n}$ are pair-wise independent random variables with $V_{n}$ being Rayleigh-distributed and $\varphi_{n}$ being uniformly distributed in the interval $[0,2 \pi$ ), (Rice, 1944, 1945). This is equivalent to saying that parameters $A_{n}$ and $B_{n}$ are normally distributed. Due to these distributions the mathematical analysis is rather complex.

The not so well know Rice's second model is the heart of the noise analysis that was presented in Sangha and Hoffmann (2005). The other way of approaching the measured noise can be given as:

$u(t)=A_{0} / 2+\sum_{n=1}^{N} A_{n} \cos \left(n \Delta \omega t+\varphi_{n}\right)$

Parameters $A_{n}$ are deterministic. Parameters $\varphi_{n}$ are random variables uniformly distributed in the interval $[0,2 \pi$ ), (Rice, 1944, 1945). Due to the uniform distributions of $\varphi_{n}$ 's, this model is mathematically much simpler than the first model.

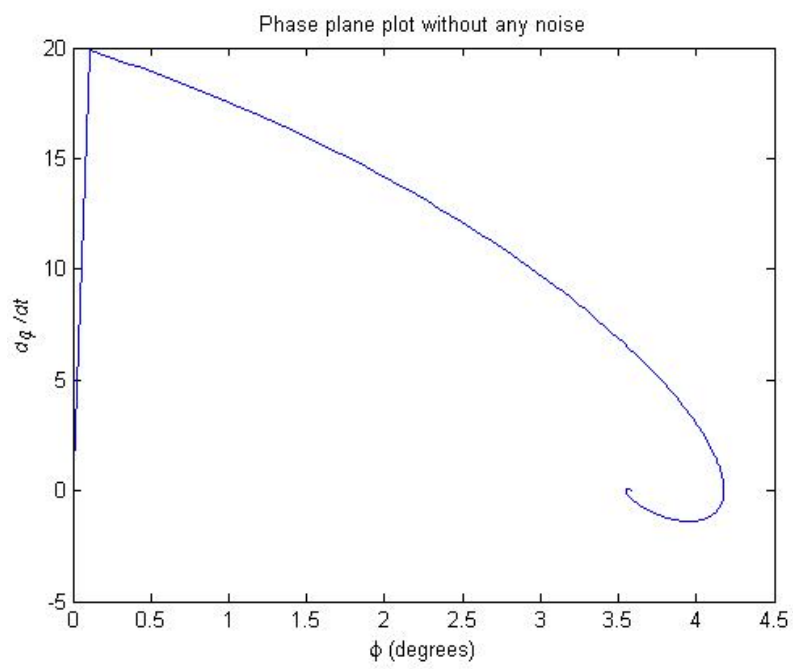

Fig. 3. Phase plane trajectory of the synthesizer without noise.

By virtue of the central limit theorem, it leads to the same results as the first model.

The accuracy of the model increases as the number $N$ of sum terms is increased. Fig. 2 demonstrates this fact by comparing the uniform distribution obtained using the Rice's second model for a value of $N$ equal to 10000 and 50000 , re- 

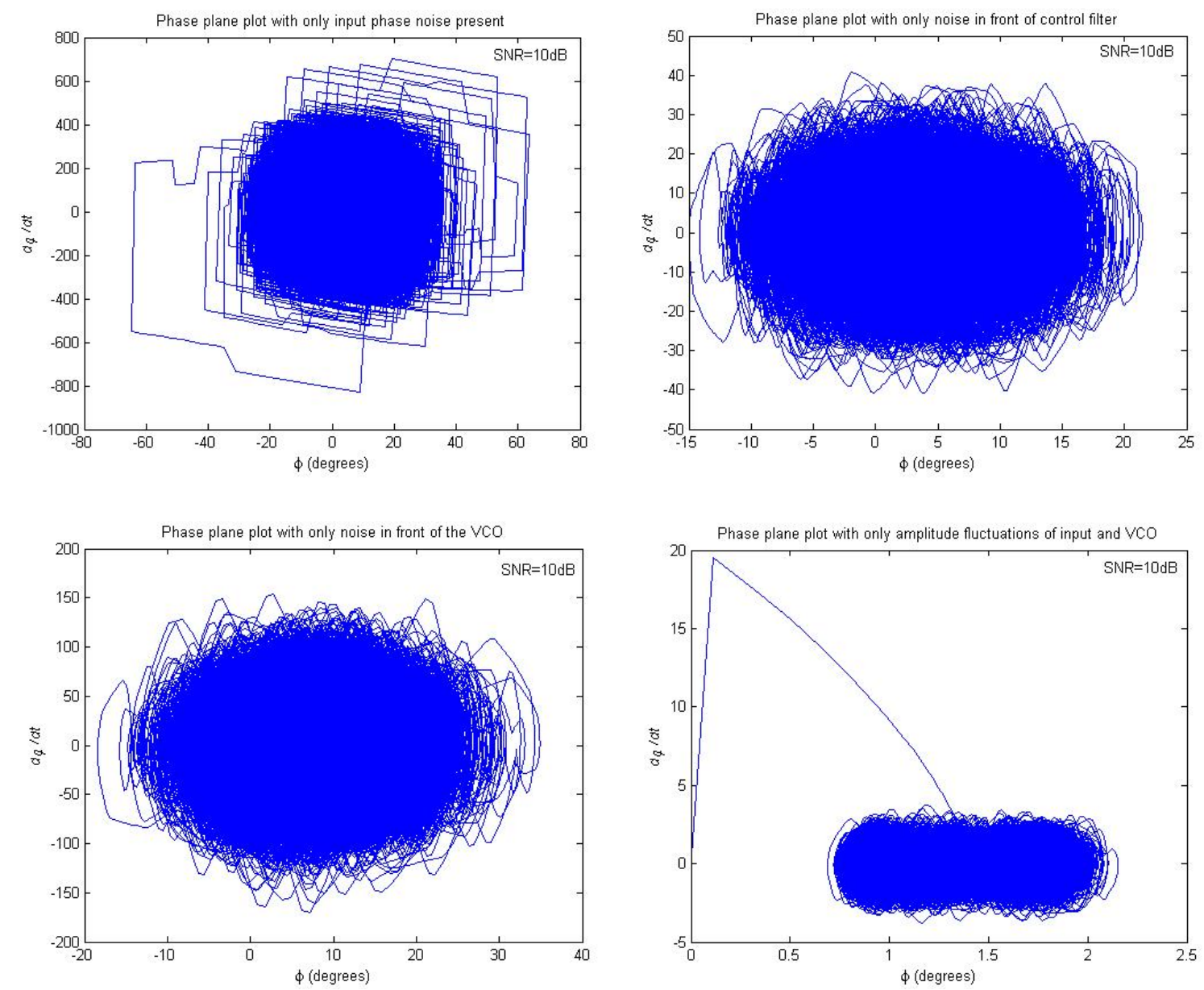

Fig. 4. Phase plane trajectories for a synthesizer with noise introduced at different points of the circuit.

spectively. The horizontal axis in this figure is divided into 50 bins in the interval $\left(-180^{\circ}, 180^{\circ}\right)$.

Note that the Rice's second model can easily be used to generate differently coloured noises. E.g. all the $A_{n}$ 's would be equal and constant for white noise. In a similar manner, $1 / f$ noise can be defined using the relation $A_{n} / \sqrt{n}$ for amplitude terms from Rice's model.

\section{Dynamic behaviour of a synthesizer with noise}

The behaviour of a PLL might not be described by a linear model when it is far from lock, for example during switching transients, and when responding to large signal inputs. Important parameters such as settling time, switching speed and capture range are often dominated by non-linear behaviour.

In Sangha and Hoffmann (2005) the behaviour of the loop with respect to noise was presented. Here we will concentrate on the nonlinear aspects of the PLL with noise. With the noise present at different points in the loop, the locking behaviour can be best demonstrated by observing the phase plane trajectories. Simulations are performed in
SIMULINK using a nonlinear model (Fig. 1) of the PLL. It does not use any of the standard VCO or PLL models found in SIMULINK.

The input noise was designed to be white noise that is divided into two components affecting the amplitude and phase respectively. The amplitude fluctuations of VCO were designed in a way that comes close to reality. Noise in front of the CF and VCO was 1/f noise superimposed by white noise.

All the random noise sources in this simulation are designed for SNR of $10 \mathrm{~dB}$. The angular frequency of the input signal is chosen to be $1.5 \cdot 10^{5} \mathrm{rad} / \mathrm{sec}$ and the damping factor $\zeta$ is chosen to be 0.7 .

Figure 3 shows the settling behaviour of the synthesizer without noise, while Fig. 4 shows how the PLL settling behaviour is modified when a noise source at one of the points shown in Fig. 1 is introduced. Comparing these curves, it is concluded that the input phase noise is most critical for the settling behaviour of the synthesizer. Furthermore, Fig. 5 shows that with increasing noise level, there is a probability of cycle slipping. Fig. 4 also shows how the amplitude fluctuations of input and VCO signals affect the settling be- 


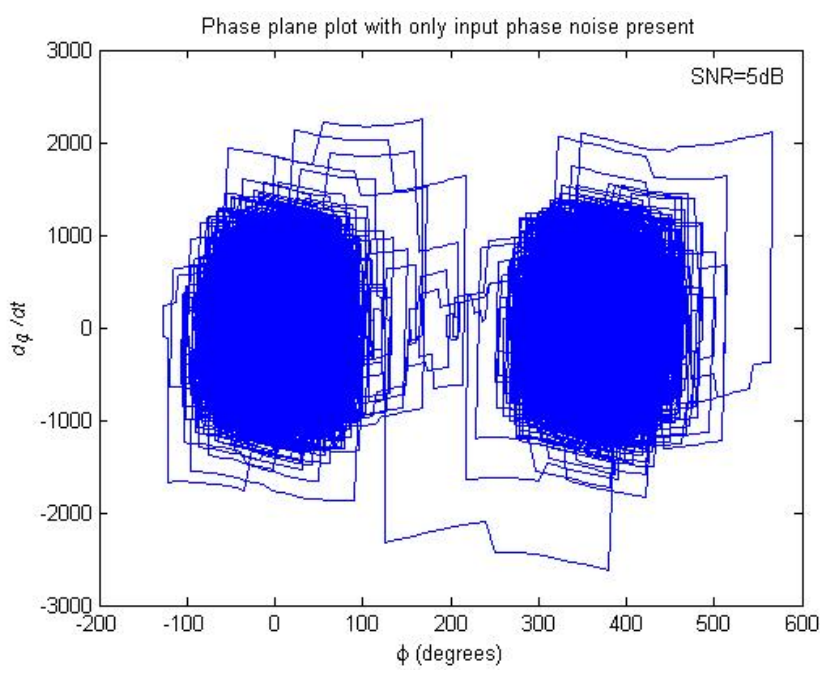

Fig. 5. Phase plane trajectory of the synthesizer with input phase noise having $\mathrm{SNR}=5 \mathrm{~dB}$.

haviour of the synthesizer. The amplitude fluctuations, which are usually ignored, are taken into account for completeness.

\section{Conclusions}

In this paper we have demonstrated a useful technique for non-linear noise analysis of a PLL based synthesizer taking into account a more complete combination of noise sources. We showed how the noise at different points in the loop can affect the settling behaviour of the synthesizer.

\section{References}

Blanchard, A.: Phase-Locked Loops Applications to Coherent Receiver Design, John Wiley and Sons, 1976.

Curtin, M. and O'Brien, P.: Phase-Locked Loops for HighFrequency Receivers and Transmitters-Part 2, Analog Devices, Analog Dialogue, 33-5, 1999.

Drucker, E.: Model PLL Dynamics and Phase-Noise Performance, Microwaves and RF Magazine, 73-82, 2000,. http://www.mwrf.com.

Heydari, P.: Analysis of the PLL Jitter Due to Power/Ground and Substrate Noise, IEEE Transactions on Circuits and Systems-I, 51, 2404-2416, 2004.

Kundert, K.: Modelling and Simulation of Jitter in PLL Frequency Synthesizers, Cadence Design Systems, Inc., 2001. www.cadence.com.

Lee, T. H. and Hajimiri, A.: Oscillator Phase Noise: A Tutorial, IEEE J. of Solid-state circuits, 35, 326-336, 2000.

Rice, S. O.: Mathematical Analysis of Random Noise, Bell Syst. Techn. J., 23, 282-332, 1944.

Rice, S. O.: Mathematical Analysis of Random Noise, Bell Syst. Techn. J., 24, 46-156, 1945.

Sancho, S. and Suàrez, A.: Nonlinear Dynamics of Microwave Synthesizers- Stability and Noise, IEEE Transactions on Microwave Theory and Techn., 49, 1792-1803, 2001.

Sangha, G. S.and Hoffmann, M. H. W.: Analysis and Design of a Frequency Synthesizer with Internal and External Noise Sources, in Proc. $18^{\text {th }}$ Intern. Conf. on Noise and Fluctuations-ICNF2005, 521-524, 2005. 